\title{
Alfvén wave evolution into magnetic filaments in 3-D space plasma
}

\author{
Horia Comişel ${ }^{1,2^{*}}$, Yasuhito Narita ${ }^{3}$ and Uwe Motschmann $n^{1,4}$
}

\begin{abstract}
Nonlinearities in space and astrophysical plasmas generate a set of magnetic filaments or bundles that have an incompressible sense of fluctuations and propagate highly obliquely to the mean magnetic field. Those filaments are caused by wave-wave interactions in which a large-amplitude Alfvén wave (or pump Alfvén wave) is disturbed by thermal, density fluctuations and collapses into obliquely propagating Alfvén waves at shorter wavelengths than the pump. Hybrid simulations confirm the filament excitations in the three-dimensional low-beta plasma. Alfvén waves develop into magnetic filaments on a time scale of about 300 ion gyrations. The filaments grow in a multi-channel way at various azimuthal angles around the mean magnetic field.
\end{abstract}

Keywords: Space plasma, Parametric instabilities, Density fluctuation

\section{Introduction}

Nonlinearities of large-amplitude Alfvén waves have been a subject of extensive studies (e.g., Hoshino and Goldstein 1989; Mjølhus and Hada 1990; Matteini et al. 2010; Dorfman and Carter 2016), since the first theoretical predictions (Sagdeev and Galeev 1969). One may immediately recognize in the MHD equations that the Lorentz force acting on the plasma parcel, $\vec{j} \times \vec{B}$ (where $\vec{j}$ is the current density and $\vec{B}$ the magnetic field), is nonlinear. Viñas and Goldstein (1991a, b) formulated the theory of parametric instabilities in two-dimensional approach using Hall MHD framework. According to this model, various kinds of wave nonlinearities are developing about the large-amplitude Alfvén wave with density perturbations depends on its direction of propagation: decay instability, modulational instability, and beat instability at low or moderate propagation angles, and magnetoacoustic (fast-mode) instability and filamentation (zerofrequency mode) instability at large propagation angles with respect to the mean magnetic field.

\footnotetext{
*Correspondence: h.comisel@tu-bs.de

${ }^{1}$ Institut für Theoretische Physik, Technische Universität Braunschweig,

Mendelssohnstraße 3, 38106 Brunswick, Germany

Full list of author information is available at the end of the article
}

Here, we address the question how large-amplitude Alfvén waves develop into the azimuthal (or transversal) plane with respect to the mean magnetic field in a threedimensional plasma. The magneto-acoustic and zerofrequency instabilities differ from the decay instability in that the pump Alfvén wave (denoted by A) interacts with the density perturbation $\mathrm{S}_{\mathrm{F}}$ and $\mathrm{S}_{\mathrm{Z}}$ with perpendicular wavevectors to the mean field and generates daughter waves $\left(\mathrm{F}^{ \pm}\right.$and $\left.\mathrm{Z}^{ \pm}\right)$in an oblique direction to the mean field. Figure 1 illustrates wavevectors and frequencies of the participating waves in the zero-frequency instability. Here, the resonant wave-wave interaction for the zero-frequency mode and fast-mode instabilities is expressed in the conventional form, $Z^{ \pm}=S_{Z} \pm A$ and $F^{ \pm}=S_{F} \pm A$, respectively. $S_{Z}$ conceptually represents a non-propagating mode. $S_{Z}$, however, may have a small but finite frequency (cf. Viñas and Goldstein 1991b).

Our three-dimensional numerical study is an extension of the hybrid plasma simulation used for testing of the three-dimensional decay instability (Comişel et al. 2019). The computational accuracies are set in favor of improving the signal-to-noise ratio by increasing the number of super-particles in the computational cell. In the actual setup, we make use of the full 3-D system (without reducing the system to a 2-D one as in the former study) 


\section{a Wavevector diagram}

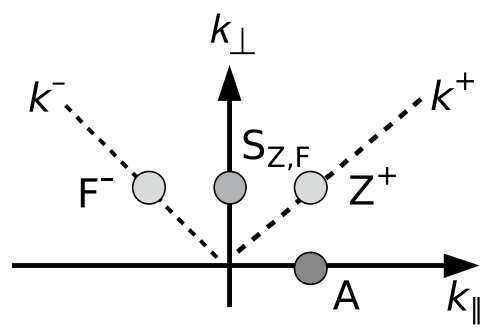

c Density perturbation dispersion

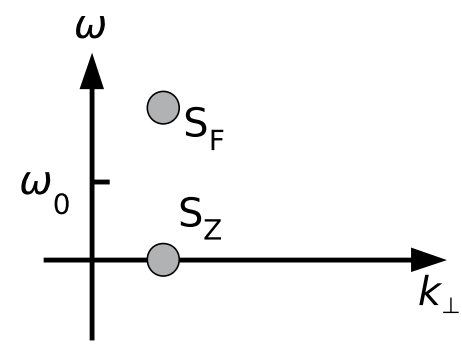

b Pump wave dispersion

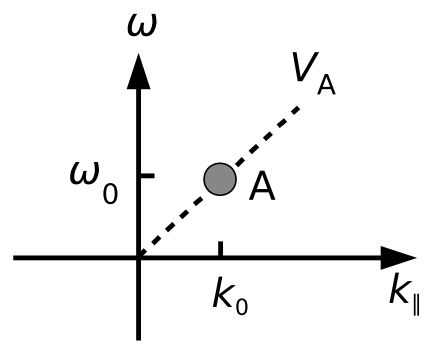

d Daughter wave dispersion

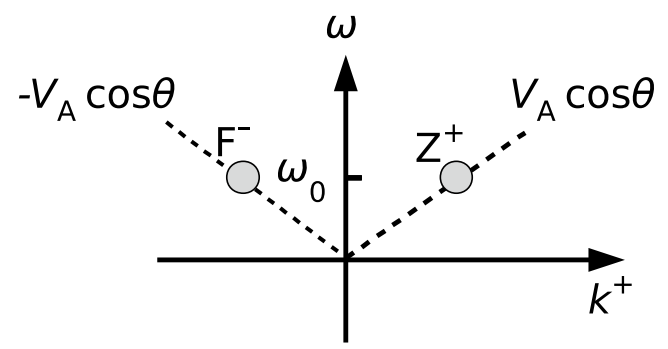

Fig. 1 Three-wave coupling of the zero-frequency and fast-mode instabilities in the wavevector domain spanning parallel and perpendicular directions (a) and in the frequency-wavenumber domain parallel (b), perpendicular (c), and oblique at $\theta$ angle (d) to the mean magnetic field

to trace the instabilities developed in the transversal (or azimuthal) plane with respect to the mean magnetic field.

The actual numerical study reveals the occurring of an instability developing in a cylindrical symmetric geometry in the azimuthal plane by forming a ring shape in the wavevector space. Here, we propose an approach to explain the observed instability in terms of the magnetoacoustic and zero-frequency perpendicular instabilities developing in the three-dimensional plasma in a roughly axial symmetric way. According to this approach, filaments develop in space and astrophysical plasmas without being wound around the mean magnetic field by fluid nonlinearity (i.e., Eddy motion).

\section{Numerical experiment using a hybrid plasma model}

Numerical experiments using a hybrid plasma code such as AIKEF (Müller et al. 2011) are particularly suited to study wave nonlinearities on the spatial scales covering both the fluid and ion-kinetic ones in two- and threedimensional space (e.g., Verscharen et al. 2012; Comişel et al. 2019). We implement the AIKEF code to a threedimensional setup with a box size of 144 ion inertial lengths in each direction. A total of $(144)^{3}$ mesh cells are used, and each cell contains 2500 super-particles at the initial time. The length scale is normalized to the ion inertial length $d_{\mathrm{i}}=V_{\mathrm{A}} / \Omega_{\mathrm{p}}$, where $V_{\mathrm{A}}$ is the Alfvén speed and $\Omega_{\mathrm{p}}$ is the ion gyro-frequency (for protons). Plasma parameter beta is set to a value of 0.01 for both ions and electrons. A moderately large-amplitude Alfvén wave is excited in the plasma as a pump with an amplitude of 0.2 to the mean field and the propagation parallel to the mean field. The pump is on the ion cyclotron branch with left-handed circular polarization around the mean field. The pump wavenumber is $k_{0} V_{\mathrm{A}} / \Omega_{\mathrm{p}}=0.21$ and the frequency $\omega_{0} / \Omega_{\mathrm{p}}=0.18$. Periodical boundary conditions are used in the simulation while we do not apply any seeds in the initial density fluctuations.

Figure 2a displays a diagram of the perpendicular fluctuation energy in magnetic field (incompressible sense) in the wavevector domain spanning the parallel and perpendicular directions to the mean magnetic field. Here, the mean magnetic field is along the $z$ direction in the real space of the reference system attached to the simulation box such that the 3-D representation of the wavevectors implies one parallel $\left(k_{\|} \equiv k_{\mathrm{z}}\right)$ and two perpendicular $\left(k_{\perp 1} \equiv k_{\mathrm{x}}, k_{\perp 2} \equiv k_{\mathrm{y}}\right)$ components. The pump Alfvén mode is identified at a parallel wavenumber of 0.21 . Figure $2 \mathrm{a}$ is a snapshot of the spectrum at a time of $t \Omega_{\mathrm{p}}=230$. At that time, the (incompressible) magnetic field fluctuations develop in the oblique directions while keeping the parallel wavenumber for the pump. Figure $2 b$ 

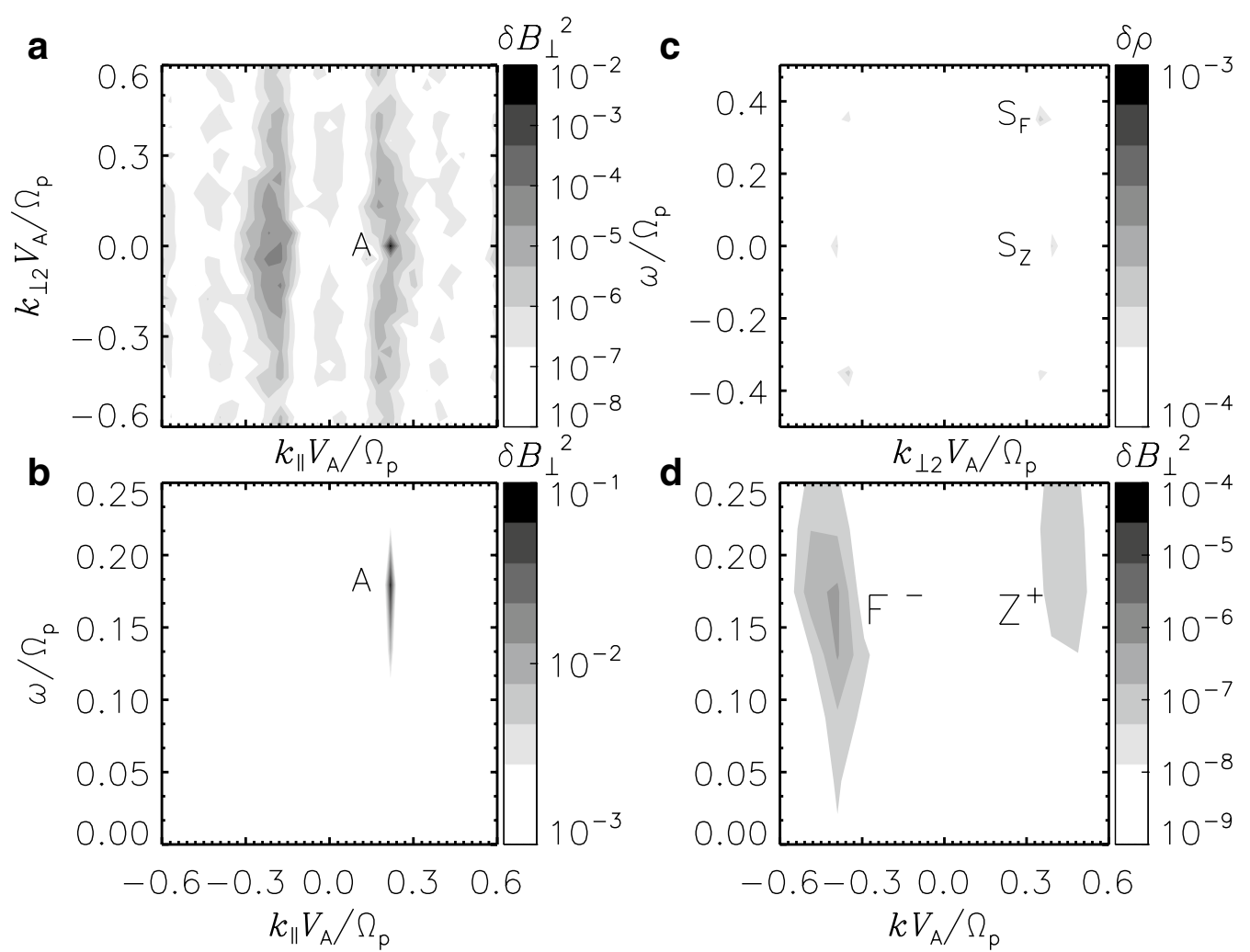

Fig. 2 Wavevector diagram for the magnetic field fluctuations (a), and dispersion diagrams for the pump Alfvén wave (b), density perturbation (c), and daughter Alfvén waves (d)

displays the dispersion diagram (energy spectrum in the $k_{\|}-\omega$ domain) for the perpendicular magnetic field fluctuations. The pump mode (labeled as "A") is identified at a wavenumber of 0.21 and a frequency of 0.18 . The dispersion diagram for the density perturbations $\mathrm{S}_{\mathrm{Z}}$ and $\mathrm{S}_{\mathrm{F}}$ which interacts with the pump generating the daughter waves $\mathrm{Z}^{+}$and $\mathrm{F}^{-}$, respectively, is displayed in Fig. 2c. Later on, we will show in Fig. 3 that the density perturbations are excited nearly axisymmetric around the mean magnetic field direction. According to Hall MHD theory (Viñas and Goldstein 1991b), the zero-frequency or the filamentation instability represents density perturbations at oblique and perpendicular propagation with small real frequencies that satisfy the condition $\operatorname{Re}(\omega) \ll \gamma$, where $\omega=\omega_{r}+i \gamma$. On the contrary, the fast mode or the magneto-acoustic instability is characterized by large real frequencies $(\operatorname{Re}(\omega) \gg \gamma)$. From this perspective, the zero-frequency density perturbation is a non-propagating spatial oscillation even if the $S_{Z}$ mode may attain finite frequencies. Finally, the dispersion diagram for the obliquely propagating daughter wave $\mathrm{Z}^{+}$ and $\mathrm{F}^{-}$is displayed in Fig. $2 \mathrm{~d}$. These modes satisfy the resonant conditions both in the frequency $\left(\omega_{\mathrm{S}_{\mathrm{Z}}}+\omega_{0}\right.$; $\left.\omega_{\mathrm{S}_{\mathrm{F}}}-\omega_{0}\right)$ and in the three-dimensional wavevector

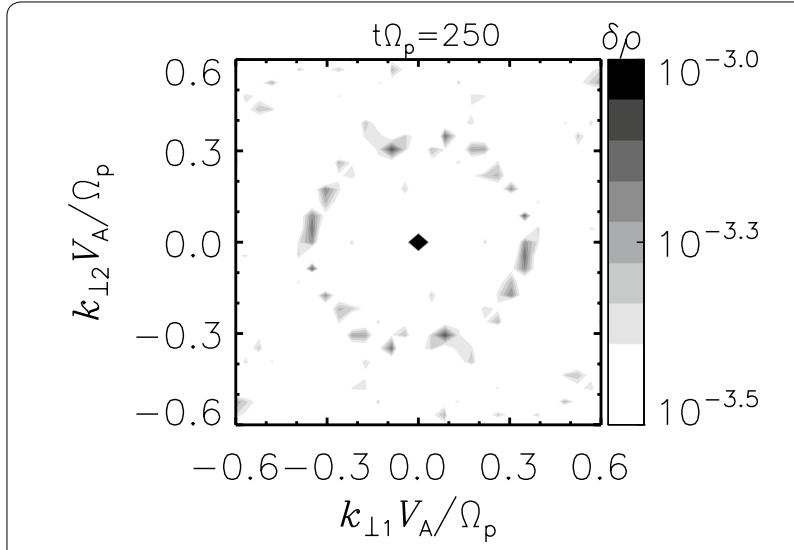

Fig. 3 Ring-shape structure generated in the density perturbation at a time of $t \Omega_{\mathrm{p}}=250$

$\left(\vec{k}_{\mathrm{S}_{\mathrm{Z}}}+\vec{k}_{0} ; \vec{k}_{\mathrm{S}}-\vec{k}_{0}\right)$. The daughter waves $\mathrm{Z}^{+}$and $\mathrm{F}^{-}$have roughly the same frequency as that of the pump (a frequency of 0.18 ) and propagate obliquely at an angle of $60^{\circ}$ to the mean field. The phase speed is consistent with that of the linear-mode Alfvén wave, $V_{\mathrm{A}} \cos \theta$ (see also the sketch in Fig. 1d). The participating waves are in all 
agreement with the three-wave coupling condition, that is $\vec{k}_{Z}^{+}=\vec{k}_{\mathrm{S}_{\mathrm{Z}}}+\vec{k}_{0},{\overrightarrow{k_{F}}}^{-}=\vec{k}_{\mathrm{S}_{\mathrm{F}}}-\vec{k}_{0}$, and $\omega_{\mathrm{Z}}^{+}=\omega_{\mathrm{Z}}+\omega_{0}$, $\omega_{\mathrm{F}}^{-}=\omega_{\mathrm{S}_{\mathrm{F}}}-\omega_{0}$, where $\omega_{\mathrm{S}_{\mathrm{Z}}} \approx 0$ and $\omega_{\mathrm{S}_{\mathrm{F}}} \approx \omega_{0}$.

Figure 3 exhibits the spectrum for the density fluctuation in the perpendicular wavevector domain at a time of $t \Omega_{\mathrm{p}}=250$. The density perturbations $\mathrm{S}_{\mathrm{Z}}$ and $\mathrm{S}_{\mathrm{F}}$ develop in the perpendicular plane into an axially symmetric way by forming a ring-shaped spectrum peaked at a wavenumber of 0.35 . The ring-shaped spectrum indicates that the zero-frequency and magneto-acoustic instabilities occur in a multi-channel fashion at various azimuthal angles simultaneously.

The time evolution diagrams for the relevant daughter modes reported in Figs. 2 and 3 are presented in Fig. 4. The modes are shown in the Fourier mode representation in the $\left(k_{\|}, k_{\perp 1}, k_{\perp 2}\right)$ wavevector domain. The field-aligned daughter modes excited by the decay instability are also reported in panels (c), (d) as well as the time evolution of the pump wave. The strengths of the daughter modes are about one order of magnitude weaker than those developed by the prominent decay instability. However, they may play a role in the dynamics of the decay instability, e.g., in its early saturation when the daughter and sound waves saturate at a low-level amplitude with respect to that one of the Alfvén pump wave (Comişel et al. 2019).

The daughter waves have normally a left-handed circular polarization according to the polarization of the Alfvén pump wave. However, we notice also a component with right-handed polarization (not shown in Fig. 2d). The analyzing of the polarization of all the excited daughter waves in our hybrid simulation is currently under study. Interesting to note is the contribution of the lower sideband $F(-5,0,8)$ which according to the coupling rules has a backward propagation similarly with the daughter waves developed by the decay instability. This study suggests that at large oblique propagation angles, the magneto-acoustic instability is responsible for generating the observed high-obliquely backward propagating daughter waves.

The dispersion analysis based on the Viñas and Goldstein (1991a) recipe provides weak growth rates for the filamentation instability at low plasma beta values in agreement with former studies. On the other hand, a much stronger magneto-acoustic instability is predicted
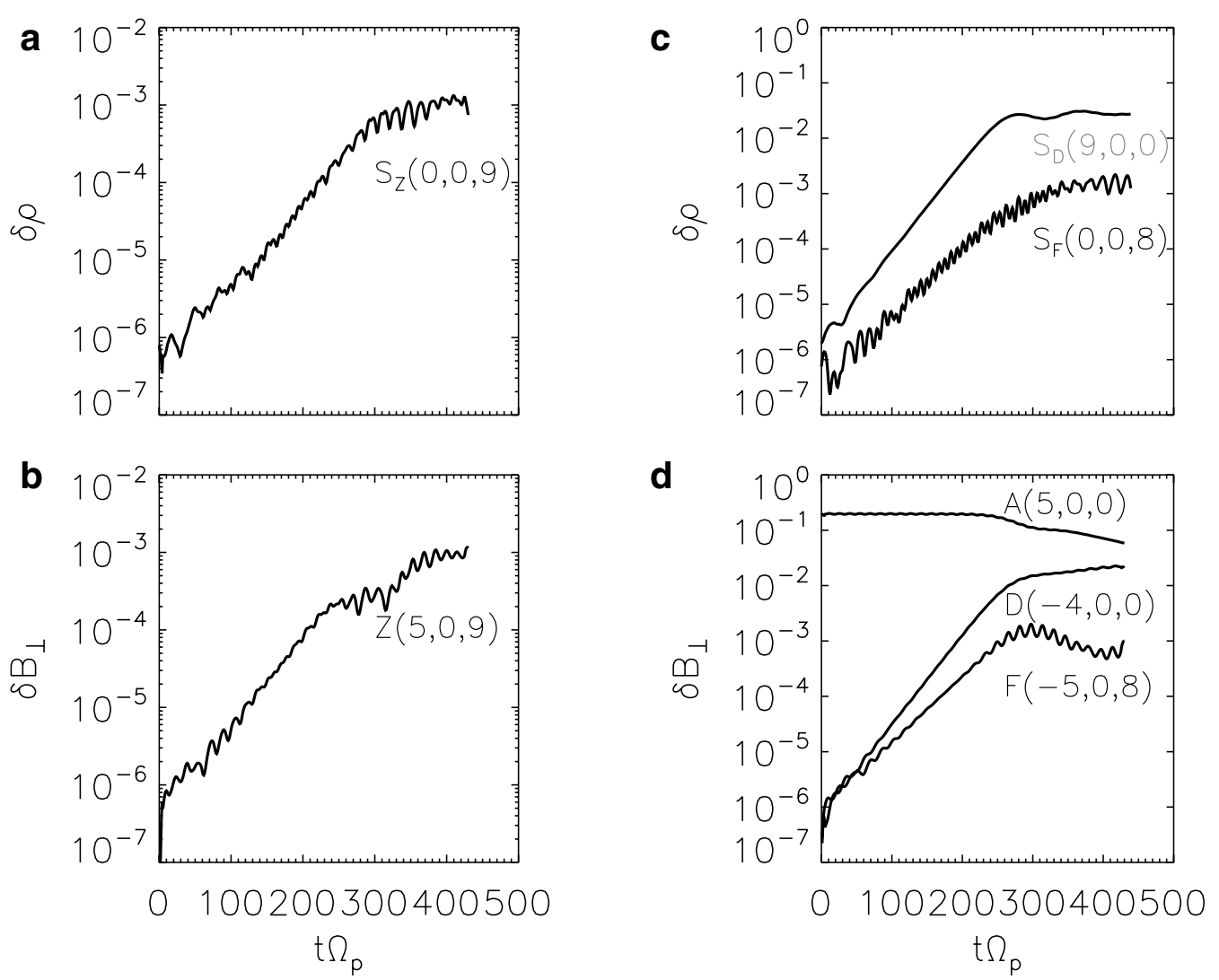

Fig. 4 Time evolution of the density and magnetic field fluctuations: longitudinal daughter mode $S_{Z}(0,0,9)$ and Alfvén sideband $Z(5,0,9)$ are shown in $\mathbf{a}$ and $\mathbf{b}$, and the longitudinal daughter mode $S_{F}(0,0,8)$ and Alfvén sideband $F(-5,0,8)$ in $\mathbf{c}$ and $\mathbf{d}$. For comparison, the Alfvén pump wave $A(5,0,0)$ and the dominant daughter modes $S_{D}(9,0,0)$ and $D(-4,0,0)$ provided by the field-aligned decay instability are also given 
in accordance with the beta dependence profile established by Viñas and Goldstein (1991b). The observed growth rates are determined from the slopes of the daughter branches given in Fig. 4. The deviation between the theoretical and the measured values of the growth rate for the fast-mode instability is within a range of $20 \%$ from the analytical estimation. The observed growth rate for the zero-frequency instability is about an order of magnitude larger than the value derived within Hall MHD model.

Concerning potential numerical artifacts, the 3-D setup would amplify a large panel of wave-wave couplings involving perpendicular modes which may or may not have physical meaning. This is one reason why we systematically studied initial physical conditions for the pump wave and plasma using many numerical setups, e.g., different values for the spatial resolution, number of super-particles, or size of the system. Overall, despite several differences noted in the time evolution of the instabilities developed by the pump wave, a ring in the wavevector domain does emerge in the transversal plane irrespective of what numerical setup is used. In low beta plasmas, the wavenumber value predicted by theory for the decay instability goes to $2 k_{0}$ when $\beta \sim 0$. Early MHD approaches (e.g., Lashmore-Davies 1976) found a singularity for the solutions of dispersion equation at $k=2 k_{0}$ and a modulational instability competing with the decay instability has been proposed. Such resonance could happen in the plasma system and thus its physical significance cannot be ignored. The 2-D Hall MHD model of Viñas and Goldstein (1991a) predicts finite growth rates spanning a circular range of radius $k=\sqrt{k_{\perp}^{2}+k_{\|}^{2}} \approx 2 k_{0}$ in the $\left(k_{\|}-k_{\perp}\right)$ wavevector domain which are decreasing with plasma beta increasing. Our simulation results suggest such a trend of instabilities radially developing in the wavevector domain. However, we agree that numerical artifacts could influence, in a certain limit, the obtained results.

\section{Summary and outlook}

A large-amplitude Alfvén wave is unstable to density perturbations or thermal fluctuations in the plasma. When wave-wave couplings occur in the three-dimensional low-beta plasma, the zero-frequency (or filamentation) mode and fast (or magneto-acoustic) mode are accompanying the decay instability.

Wave-wave interactions occur in a multi-channel way in the three-dimensional plasma. The hybrid plasma simulation study identified that the decay instability develops not only in the parallel direction to the mean magnetic field but also obliquely, forming multi-channel couplings (Comişel et al. 2019). The filamentation and magnetoacoustic processes in the three-dimensional plasma also develop in a multi-channel way, that is, the density perturbation and daughter waves grow at various azimuthal angles in the perpendicular plane.

To conclude, we note that in the three-dimensional plasmas all (or nearly all) the possible wave-wave couplings are realized in a multi-channel way; and this happens both in the parallel-perpendicular domain (which is the case for the decay instability) and in the azimuthal angle sense (which is the case for the zero-frequency and magneto-acoustic instabilities discussed in the paper). The multi-channel possibility has so far largely been overlooked because the analytic theory typically deals with a wave-wave coupling in a particular set of propagation directions (of the pump or density perturbation) and the earlier numerical simulations were limited to lower dimensions (1-D or 2-D). Azimuthal degree of freedom should not be neglected when studying the wave-wave coupling in three dimensions.

Space missions, e.g., Parker Solar Probe, would detect the on-going linear or further nonlinear filamentation closest at 10 solar radii or larger distance from the Sun. Regarding the astrophysical implication, solar wind turbulence is known to be axially asymmetric around the mean magnetic field, both in the wavevector spectrum and in the fluctuation directions (e.g., Narita et al. 2010; Roberts et al. 2017). In our numerical experiment, the breakdown of axial symmetry cannot be explained as a fundamental nature of the filamentation (or magnetoacoustic) instability. The axial asymmetry in solar wind turbulence must have an origin other than the intrinsic wave-wave interactions in plasmas. Asymmetric flow expansion among radial and non-radial directions from the Sun might be one of the mechanisms.

\section{Acknowledgements \\ YN is grateful to Masahiro Hoshino and staff in his group at the University of Tokyo for their hospitality and discussion during the research visit, which was supported by the Japan Society for the Promotion of Science, Invitational Fellowship for Research in Japan (Long-term) under grant FY2019 L19527. We acknowledge support by the German Research Foundation and the Open Access Publication Funds of the Technical University of Braunschweig.}

\section{Authors' contributions}

HC worked on theory development, simulations, and manuscript writing. YN worked on modeling of wave nonlinearities, applications to space plasmas, and manuscript writing. UM worked on theory development, discussion, and supervision of the study. All authors read and approved the final manuscript.

\section{Funding}

The work by $\mathrm{HC}$ is financially supported by a grant of the Deutsche Forschungsgemainschaft under contract DFG MO539/20-1. The authors gratefully acknowledge the Gauss Centre for Supercomputing e.V. (http://www.gauss -centre.eu) for funding this project by providing computing time through the John von Neumann Institute for Computing (NIC) on the GCS Supercomputer JUWELS at Juelich Supercomputing Centre (JSC). The work by YN is financially supported by the Austrian Space Applications Programme (ASAP) at the Austrian Research Promotion Agency, FFG ASAP12 SOPHIE, under contract 853994. The work by HC in Bucharest is supported by ESA project MAGICS, PRODEX contract C4000127660. This open-access publication was funded by Technische Universität Braunschweig. 
Data availability statement

Simulation data can be provided upon request.

\section{Competing interests}

The authors declare that they have no competing interests.

\section{Author details}

${ }^{1}$ Institut für Theoretische Physik, Technische Universität Braunschweig, Mendelssohnstraße 3, 38106 Brunswick, Germany. ${ }^{2}$ Institute for Space Sciences, P.O. Box MG-23, Atomiştilor 409, 077125 Bucharest-Măgurele, Romania. ${ }^{3}$ Space Research Institute, Austrian Academy of Sciences, Schmiedlstraße 6, 8042 Graz, Austria. ${ }^{4}$ Deutsches Zentrum für Luft- und Raumfahrt, Institut für Planetenforschung, Rutherfordstraße 2, 12489 Berlin, Germany.

Received: 31 October 2019 Accepted: 24 February 2020

Published online: 05 March 2020

\section{References}

Comişel H, Narita Y, Motschmann U (2019) Multi-channel coupling of decay instability in three-dimensional low-beta plasma. Ann Geophys 37:835-842. https://doi.org/10.5194/angeo-37-835-2019

Dorfman S, Carter TA (2016) Observation of an Alfvén wave parametric instability in a laboratory plasma. Phys Rev Lett 116:195002. https://doi. org/10.1103/PhysRevLett.116.195002

Hoshino H, Goldstein ML (1989) Time evolution from linear to nonlinear stages in magnetohydrodynamic parametric instabilities. Phys Fluids B Plasma Phys 1:1405-1415. https://doi.org/10.1063/1.858971

Lashmore-Davies CN (1976) Modulational instability of a finite amplitude Alfvén wave. Phys Fluids 19:587. https://doi.org/10.1063/1.861493

Matteini L, Landi S, Del Zanna L, Velli M, Hellinger P (2010) Parametric decay of linearly polarized shear Alfvén waves in oblique propagation: one and two-dimensional hybrid simulations. Geophys Res Lett 37:L20101. https ://doi.org/10.1029/2010GL044806

Mjølhus E, Hada T (1990) Oblique stability of circularly polarized MHD waves. J Plasma Phys 43:257-268. https://doi.org/10.1017/S002237780001477X

Müller J, Simon S, Motschmann U, Schüle J, Glassmeier K-H, Pringle GJ (2011) A.I.K.E.F.: adaptive hybrid model for space plasma simulations. Comp Phys Commun 182:946-966. https://doi.org/10.1016/j.cpc.2010.12.033

Narita Y, Glassmeier K-H, Sahraoui F, Goldstein ML (2010) Wave-vector dependence of magnetic-turbulence spectra in the solar wind. Phys Rev Lett 104:171101. https://doi.org/10.1103/PhysRevLett.104.171101

Roberts OW, Narita Y, Escoubet CP (2017) Direct measurement of anisotropic and asymmetric wave vector in ion-scale solar wind turbulence. Astrophys J Lett 851:L611. https://doi.org/10.3847/2041-8213/aa9bf3

Sagdeev RZ, Galeev AA (1969) Nonlinear plasma theory. Benjamin, New York Verscharen D, Marsch E, Motschmann U, Müller J (2012) Parametric decay of oblique Alfvén waves in two-dimensional hybrid simulations. Phys Rev E 86:027401. https://doi.org/10.1103/PhysRevE.86.027401

Viñas AF, Goldstein ML (1991a) Parametric instabilities of circularly polarized large-amplitude dispersive Alfvén waves: excitation of parallel-propagating electromagnetic daughter waves. J Plasma Phys 46:107-127. https:// doi.org/10.1017/S0022377800015981

Viñas AF, Goldstein ML (1991b) Parametric instabilities of circularly polarized large-amplitude dispersive Alfvén waves: excitation of obliquely-propagating daughter and side-band waves. J Plasma Phys 46:129-152. https:// doi.org/10.1017/S0022377800015993

\section{Publisher's Note}

Springer Nature remains neutral with regard to jurisdictional claims in published maps and institutional affiliations.

\section{Submit your manuscript to a SpringerOpen ${ }^{\circ}$ journal and benefit from:}

- Convenient online submission

- Rigorous peer review

- Open access: articles freely available online

- High visibility within the field

- Retaining the copyright to your article

Submit your next manuscript at $\boldsymbol{\nabla}$ springeropen.com 